\title{
Efficient design method of segmented lenses for lighting applications with prescribed intensity and low peak luminance
}

\author{
March 27, 2018
}

\begin{abstract}
LEDs that are used today in general lighting applications provide high luminous flux from small packages which can result into high peak luminance and glare. The use of diffusors allows to reduce peak luminances, but these components do not allow accurate control over the resulting intensity pattern as is possible with non-imaging lenses for low étendue sources such as LEDs. In this paper a new design method is presented to create a rotational or transverse symmetric segmented lens which results in a reduced peak luminance but still allows excellent control over the resulting intensity distribution. Each segment of the lens spreads out the light in a certain angular range, but the different contributions of each lens segment result together into the desired target intensity distribution. The design method is based on the iterative optimization of a linear transformation of a source intensity distribution into a target intensity distribution, starting from a transformation that yields maximal light beam spreading.
\end{abstract}

\section{Introduction}

The typical purpose of lenses in lighting applications is to transform the light distribution generated by the light source, typically an LED nowadays, into a new light distribution with a specific intensity. A very accurate prescribed intensity distribution can be generated by a freeform lens in combination with a point source. For a 'conventional' freeform lens, the transformation of the light distribution is a one-to-one mapping of rays from entrance to output [Bruneton et al., 2013a; Feng et al., 2016]. In the $2 \mathrm{D}$ case considered in this paper a mapping can simply be found by matching the cumulative distributions of the source and target distributions. This approach offers accurate control over the resulting intensity/illuminance but no control over the resulting luminance distribution at the output of the lens.

In the domain of lighting there is still a lot of ongoing research on the topics of visual discomfort and glare [Geerdinck et al., 2014; Scheir et al., 2015; Tashiro et al., 2015], but a recurring theme in all models is that visual discomfort is strongly correlated to both the peak luminance and the homogeneity of the luminance distribution of the luminaire. When a point-like source, such as an LED, is observed through a smooth lens, the observed peak luminance will be the same as the observed peak luminance without the lens. Currently, the main method to reduce the observed peak luminance is by introducing a volume or surface scattering diffuser in front of the light source [Helm and Benoit, 1990; Rinehart et al., 2015; Yamamoto and Akao, 2012]. Although effective, the reduction in the observed peak luminance comes at the cost of a loss of accurate control over the final resulting intensity pattern.

This paper describes a new design method to create a segmented lens that spreads out the light that is going in each direction over several lens segments while still maintaining good control 
over the resulting intensity pattern. When the lens segments are sufficiently small, this approach effectively reduces the observed peak luminance and improves the overall homogeneity of the resulting light distribution. This will result in an increased visual comfort. All lens segments redistribute the incident light towards well-tuned, overlapping angular ranges in such a way that the targeted intensity pattern is achieved. The design method uses a point source approximation for the light source as the method is specifically intended for LEDs with a small spatial extent. With such LEDs a very precise tailoring of the intensity distribution can be realized. With extended sources such as multi-die LED packages the tailoring of the intensity distribution will be much less accurate due to the higher tendue.

The method is currently limited to the design of two dimensional lens contours that can be extruded or rotated to obtain realistic 3D segmented lenses.

Section 2 of this paper describes the design method of the 2D lens contour and details the underlying concepts. In section 3 the method is then illustrated for the case of a rotationally symmetric segmented 3D lens that generates a circular pattern.

\section{Method}

\subsection{Goals of the design}

The considered optical configuration is a point source that is encompassed by a $2 \mathrm{D}$ lens structure as is illustrated in Fig. 1. The inner surface of this lens structure is a circle with the point source at the center. The outer surface is an optically active surface that consists of multiple refractive segments positioned at the same radial distance from the source. The refracted light from the light source at each segment is spread out in such a way that the output light distributions from neighbouring lens segments overlap as much as possible in the far-field and result all together in the desired intensity pattern. The method to be discussed only generates the segmented outer surface. Therefore, a half circle is chosen as the inner surface so that refraction only happens at the outer surface. The method could be expanded to any other lens shape with the added complexity of taking the refraction at the first lens surface into account.

The goal of the design method is thus to achieve a segmented optical surface that transforms the intensity distribution $S(\alpha)$ from the light source into a target far-field intensity distribution $T(\beta)$ with $\alpha$ and $\beta$ the angular coordinates of both intensity distributions. As is schematically represented in Fig. 2. In order to reduce the observed peak luminance from all observer positions it is crucial that the light is spread out as much as possible at each segment. The maximum angle over which the incoming rays can be refracted is chosen to be the deviation angle $B$ that corresponds with an angle of incidence on the segmented surface that is equal to the Brewster angle. When rays would be refracted over larger angles than this maximum deviation angle $B$, Fresnel reflections would increase considerably resulting in a lower optical efficiency and/or a high amount of stray light that can distort the target intensity distribution.

With this limitation in consideration, for a circular lens it is only possible to transform a source distribution $S(\alpha)$ into a target distribution $T(\beta)$ only if for each $\alpha^{\prime}$ there exist a $\beta^{\prime} \in\left[\alpha^{\prime}-B, \alpha^{\prime}+B\right]$ such that:

$$
\int_{0}^{\alpha^{\prime}} S(\alpha) \mathrm{d} \alpha=\int_{0}^{\beta^{\prime}} T(\beta) \mathrm{d} \beta
$$

If this requirement is not met, the target intensity distribution cannot be obtained with a circular lens. In this case additional shaping of the light beam is necessary, either at the inner surface of the 


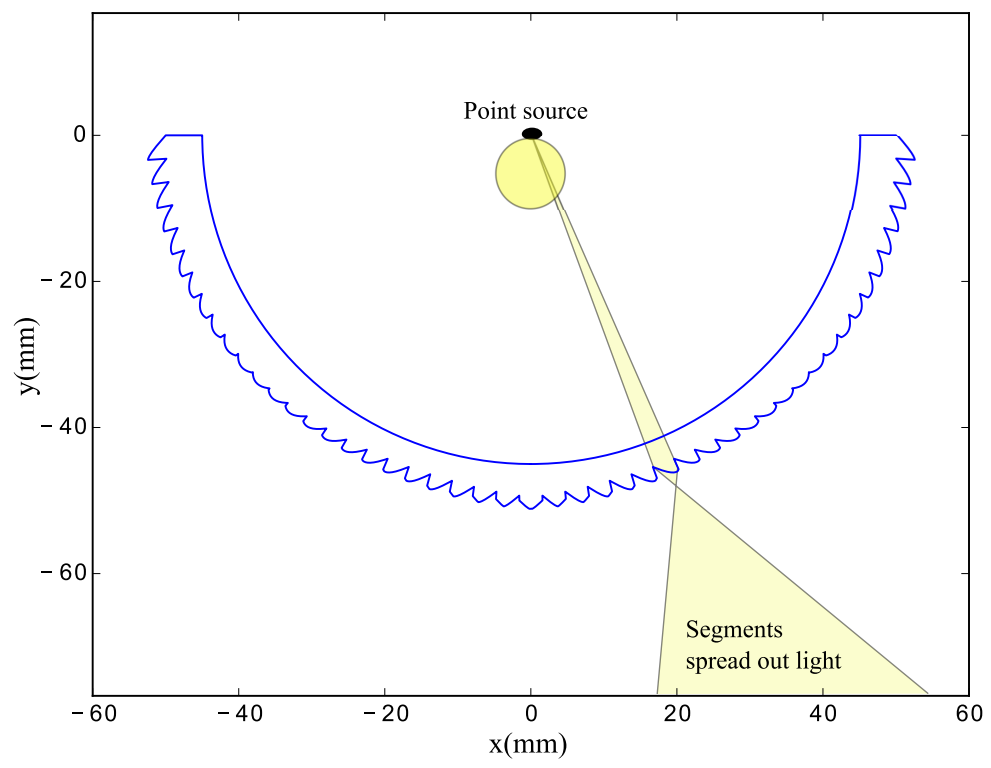

Figure 1: Example of the 2D lens contours of a segmented lens.

lens or with a separate optical component such as a primary lens closer to the light source. This extra refraction will alter the source distribution $S$. As long as the refracted rays do not cross each other, this new distribution can be used as the source distribution, by taking into account the new ingoing ray directions.

In the following section, the design process will be illustrated for the 2D example of transforming the 2D Lambertian intensity distribution $S(\alpha)=\cos (\alpha)$ into a two lobed target distribution $T(\beta)=$ $\sin (2 \beta)$ with $\alpha$ and $\beta$ defined over the range $\left[-\frac{\pi}{2}, \frac{\pi}{2}\right]$.

The segmented lens encompasses the full angular light distribution $S(\theta)$ of the light source. For every angle $\alpha$ the light is redistributed by the segmented lens according to a specific 1-dimensional distribution $M(\alpha, \beta)$. The challenge is to find and tune the distributions for every $\alpha$ so that combined they form the target distribution $T(\beta)$. In other words, a 2-dimensional linear transformation $M(\alpha, \beta)$ has to be found that transforms the source distribution $S(\alpha)$ into the target distribution $T(\beta)$ according to

$$
T(\beta)=\int M(\alpha, \beta) S(\alpha) \mathrm{d} \alpha
$$

When the requirement (1) is met, there is an infinite set of linear transformations that satisfy this equation. The most obvious example of such a transformation is the ray-to-ray mapping that results in a smooth 2D freeform lens surface. However, to reach the goal of a reduced peak luminance with a segmented lens that can be fabricated, the transformation should satisfy the following conditions:

1. The transformation fully utilizes the 'Brewster angle range' to spread out the light in each point. Mathematically this comes down to $\forall \alpha^{\prime}: \forall \beta \in\left[\alpha^{\prime}-B, \alpha^{\prime}+B\right]: M\left(\alpha^{\prime}, \beta\right) \neq 0$ unless $T(\beta)=0$, with $B$ again the maximum deviation angle that corresponds with a ray that has an angle of incidence equal to the Brewster angle. 


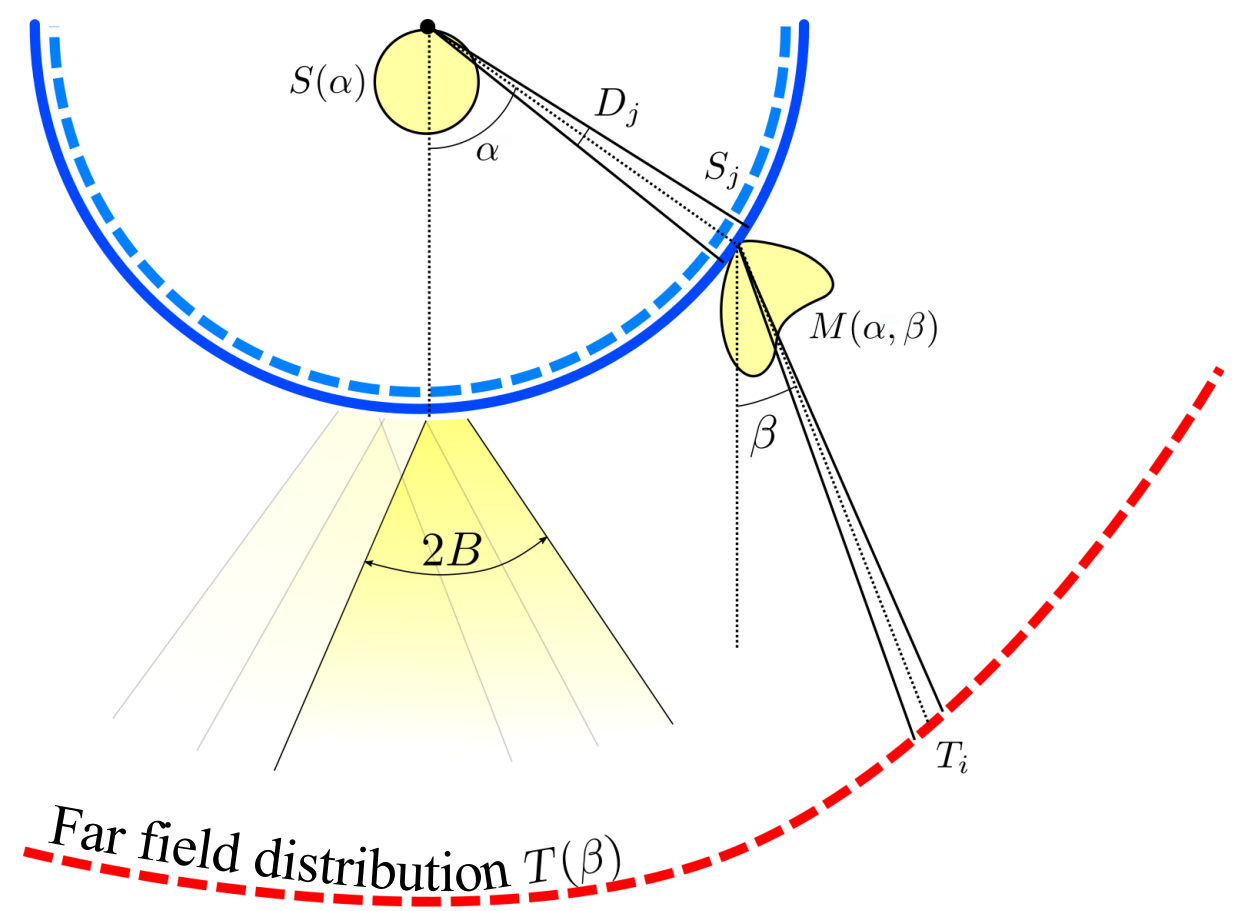

Figure 2: At every segment $j$ that spans an angular domain $D_{j}$ of the segmented lens surface, a light flux $S_{j}$ is spread out over an angular range that is two times the maximum deviation angle $B$ with a distribution $M(\alpha, \beta)$. All overlapping light contributions from the different segments together result in the target distribution $T(\beta)$.

2. In addition, each distribution $M\left(\alpha^{\prime}, \beta\right)$ should be continuous such that the resulting lens segments are smooth curved curves.

As this concerns a segmented lens with discrete structures, the problem can be discretized.

The source distribution $S(\alpha)$ and the outer lens surface are angularly divided into $s$ equal parts, each corresponding with a single lens segment. The light flux $S_{j}$ coming from the source and going through section $j$ of the segmented lens surface is:

$$
S_{j}=\int_{D_{j}} S(\alpha) \mathrm{d} \alpha
$$

wherein $D_{j}$ is the angular domain of section $j$ on the lens. This is also illustrated in Fig. 2

Similarly, the angular domain of the target distribution $T(\beta)$ is divided into $t$ equal sections. The total desired light flux in section $i$ is then denoted by $T_{i}$. As the division of the target distribution is not related with the number of lens segments, the number $t$ can be chosen according to the desired resolution of the target distribution. In general $t \gg s$.

The problem of finding a suitable $2 D$-function $M(\alpha, \beta)$ is then reduced to finding a matrix $M$ that transforms the vector $\mathbf{S}=\left[S_{0} \ldots S_{s}\right]^{\top}$ representing the source distribution into the vector $\mathbf{T}=\left[T_{0} \ldots T_{t}\right]^{\top}$ representing the target distribution. 


$$
\left[\begin{array}{c}
T_{0} \\
\vdots \\
T_{t}
\end{array}\right]=\left[\begin{array}{ccc}
m_{00} & \cdots & m_{0 s} \\
\vdots & \ddots & \vdots \\
m_{t 0} & \cdots & m_{t s}
\end{array}\right]\left[\begin{array}{c}
S_{0} \\
\vdots \\
S_{s}
\end{array}\right]
$$

To find a transformation matrix $\mathrm{M}$ that satisfies Eq. (4) and complies with the desired conditions for the ray transformation stated above, an iterative approach is used. An initial matrix $M^{0}$ is plugged into Eq. (4) and the resulting difference with the target distribution is used as feedback to modify the transformation matrix.

To construct the initial matrix $M^{0}$, it is assumed that the transformation $M^{0}$ spreads the light from the source emitted in each direction $\alpha$ uniformly over the range $[\alpha-B, \alpha+B] . M^{0}$ is thus a sparse matrix with only non-zero elements within a constant vertical range of the matrix diagonal. In general the transformation matrix is not square, so the diagonal is the collection of elements that represent the angular domains that contain $\alpha=\beta$. Since each column of $M^{0}$ represents the light distribution from the corresponding lens segment and it is assumed that the incident light flux is conserved, all columns of the matrix $M^{0}$ are normalized. A visual representation of the matrix $M^{0}$ is shown in Fig. 3

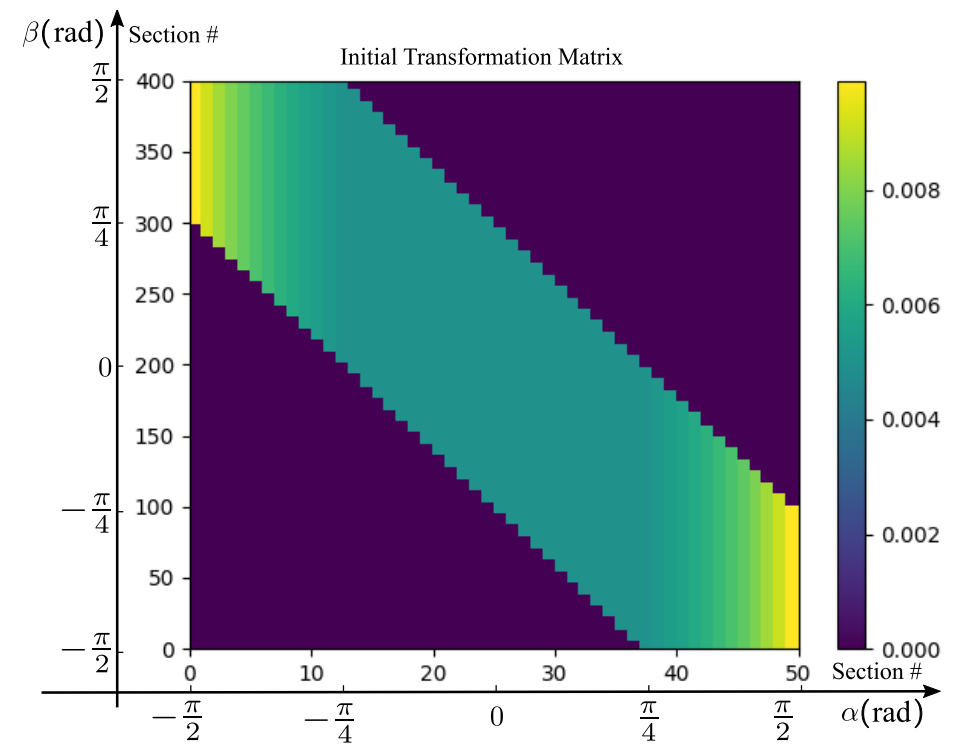

Figure 3: Visual representation of the initial matrix $M^{0}$ for a lens with 50 segments and 400 target bins.

The initial matrix $M^{0}$ is subsequently used to mix up the light from the source distribution $\mathbf{S}$ to a temporary target distribution $\mathbf{T}^{0}$.

$$
\mathbf{T}^{0}=M^{0} \mathbf{S}
$$

The resulting light distribution $\mathbf{T}^{0}$, which can be seen as the green line in Fig. 4 for the example of a Lambertian source distribution and a double-lobed target distribution, will typically differ from the desired target distribution $\mathbf{T}$. Therefore, it is necessary to adapt the matrix $M^{0}$. This is done 
by adapting the outgoing distributions of the lens segments which are represented by the columns of the matrix $M^{0}$.

For each column $j$, each element $i$ is rescaled by the ratio of the desired target value $T_{i}$ to the obtained target value $T_{i}^{0}$ and the column is subsequently renormalised. The normalisation of the columns is necessary to guarantee conservation of energy at each lens segment. The resulting transformation matrix is used to calculate a new intermediate target distribution $\mathbf{T}^{1}$ after which the same procedure can be repeated. This results in a three-step process that is repeated until $\mathbf{T}^{n}$ sufficiently approaches $\mathbf{T}$.

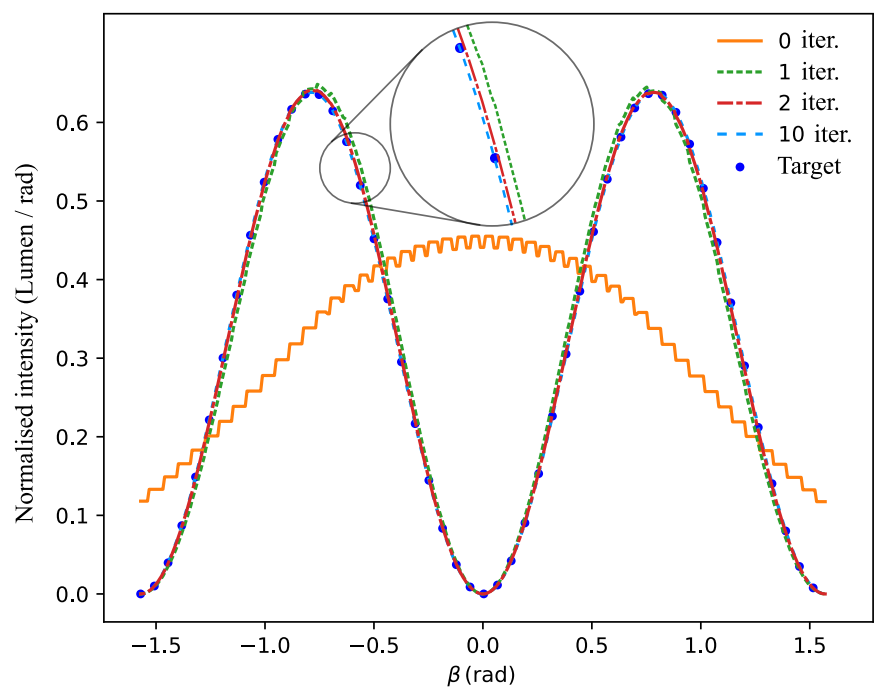

Figure 4: The obtained intermediate target distributions $\mathbf{T}^{k}$ can be seen for iterations $k=0,1,2$ and 10.

1. Calculate the temporary target distribution $\mathbf{T}^{k}$

$$
\mathbf{T}^{k}=M^{k} \mathbf{S}
$$

2. Adjust the transformation matrix to the target $\mathbf{T}$

$$
M_{i j}^{k+1}=M_{i j}^{k} \frac{T_{i}}{T_{i}^{k}}
$$

3. Normalize each column $M_{j}^{k+1}$

4. Calculate the new intermediate target distribution $\mathbf{T}^{k+1}$

$$
\mathbf{T}^{k+1}=M^{k+1} \mathbf{S}
$$

If $\left\|\mathbf{T}^{k+1}-\mathbf{T}\right\|<\epsilon$ (with $\epsilon$ arbitrarily small) the algorithm ends and $M^{k+1}$ is the optimized matrix; otherwise go back to step 2 . 
The shape of the initial matrix is conserved with this scheme; in each iteration step every element of the matrix is multiplied by a factor which means that zero elements remain zero elements. This guarantees that the light deflection at each lens segment does not exceed the maximum deviation angle $B$. In each iteration, the columns of the matrix are normalized and therefore the solution is guaranteed to never diverge. If the source and target distributions $S(\alpha)$ and $T(\beta)$ satisfy Eq. (1), the algorithm will converge towards a transformation matrix that transforms $\mathbf{S}$ into $\mathbf{T}$ which is illustrated in Fig. 4 for $S(\alpha)=\cos (\alpha)$ and $T(\beta)=\sin (2 \beta)$. The corresponding final transformation matrix $M$ is illustrated in Fig. 5 .

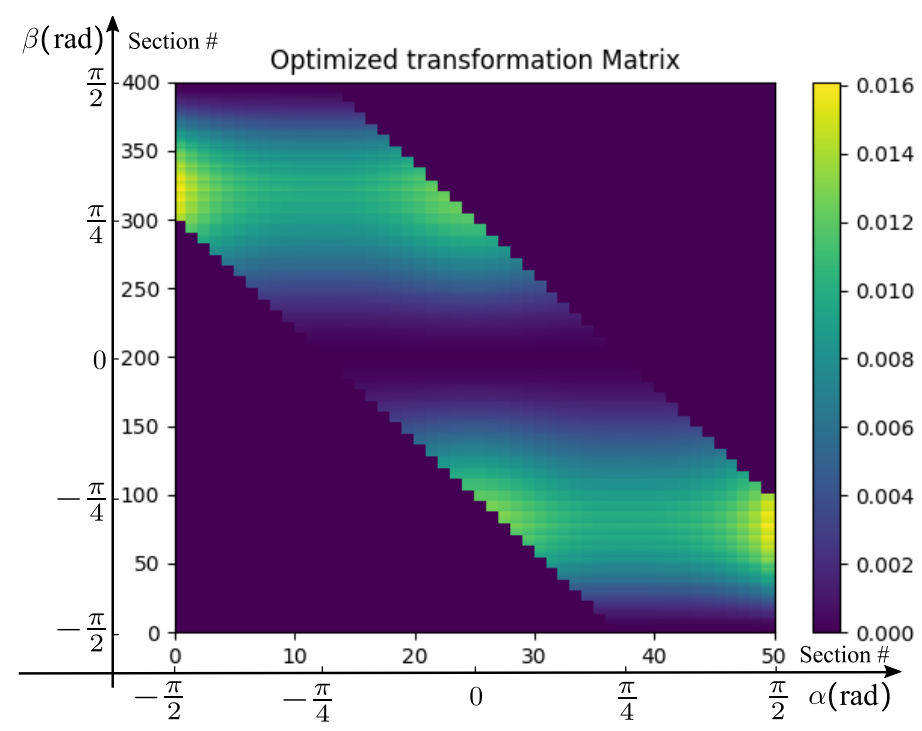

Figure 5: The matrix $M$ after 10 iterations of optimization. Each column represents the light distribution exiting from the corresponding lens segment.

\subsection{Reduction of the peak luminance}

If each column ' $j$ ' $M_{j}$ of the final transformation matrix $M$ is now multiplied with the corresponding light flux $S_{j}$ coming from the source and going through section $j$ of the segmented lens surface, one obtains a new matrix $F$ where each matrix element $F_{i j}$ represents the fraction of the total emitted light that comes from a certain lens segment $j$ and goes in a certain direction $\left[\beta_{i}-\Delta \beta, \beta_{i}+\Delta \beta\right]$. This matrix F, for the same example as used for figures 4 and 5 , is illustrated in Fig. 6 .

Each row $F_{i}$ of this matrix $F$ thus represents the distribution of light flux emitted in a direction $\left[\beta_{i}-\Delta \beta, \beta_{i}+\Delta \beta\right]$ across all the segments of the lens surface.

If the light source is a point source, each element of the row $F_{i}$ that corresponds to an angle $\beta_{i}$ is proportional to the observed luminance at the segmented lens surface when viewed from the direction $\beta_{i}$. Therefore, the peak luminance is proportional to the largest light flux emitted from a certain lens segment in that direction given by the maximal value of the row $F_{i}$. This assumes that the lens segments are comparable or larger than the eye resolution spot of the observer at the lens surface. When this is not the case, the flux distribution given by a row $F_{i}$ should be convoluted with the eye resolution spot in order to be proportional to the observed luminance. 
If the lens would have a smooth non-segmented freeform surface, the light flux emitted in each direction would be the same but would come from a single point on the lens surface instead of multiple points as is the case with a segmented lens surface. Therefore, the effective relative peak luminance reduction by a segmented lens compared to the case of a non-segmented lens at a certain observation angle $\beta_{i}$ is equal to the fraction of the largest light flux from a lens segment in that direction (given by the maximal value of row $F_{i}$ ) over the total light flux emitted in that direction (given by the sum of all values of row $F_{i}$ ). A plot of this luminance reduction factor in each direction $\beta$ for a lens with 50 structures is plotted in Fig. 7. It can be seen that compared to a smooth freeform surface, the peak luminance can be reduced down to a factor $1 / 20$ in some directions.

We remark that the effective peak luminance reduction in a practical situation will depend on the size of the used light source and the size of the eye resolution spot of the observer compared to the size of the lens segments. A detailed analysis of this effective peak luminance reduction is outside the scope of this publication where the focus is on the design algorithm. Another discussion point could be the question if the presented iterative algorithm always converges to the transformation matrix that corresponds with the highest peak luminance reduction for all observer directions. Although we cannot mathematically prove this at this moment; by using a starting matrix $M_{0}$ that spreads the light from the source uniformly over the maximum deflection angle, the method always converges to a very good solution for all practical cases that we have already tested.

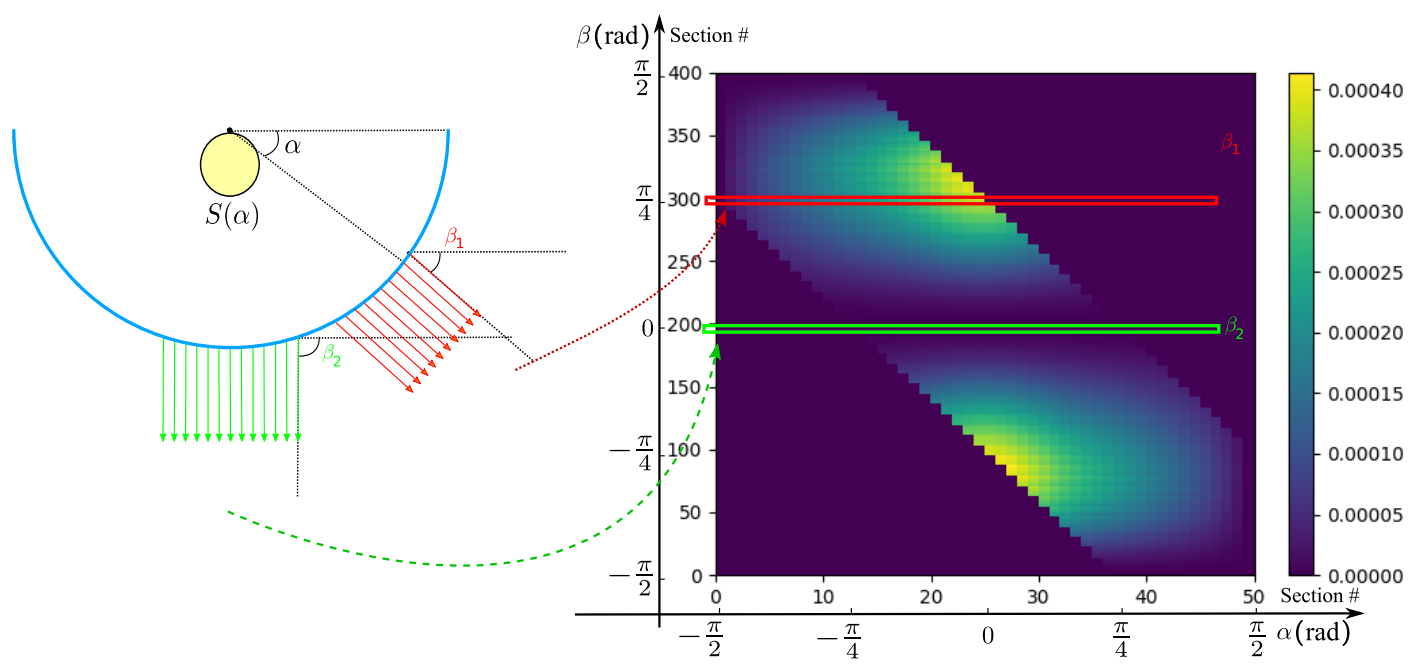

Figure 6: The matrix $F$ where each element $F_{i j}$ represents the fraction of the total light that comes from a certain lens element $j$ and goes in a certain direction $\left[\beta_{i}-\Delta \beta, \beta_{i}+\Delta \beta\right]$. Assuming a point source, each row is directly proportional to the observed luminance distribution across the lens surface when viewed from an angle $\beta_{i}$

\subsection{Calculation of the segmented lens surface}

When a sufficiently optimized transformation matrix $M$ has been found, the surface of each lens segment $j$ can be derived from the input distribution $S(\alpha)$ and the column $M_{j}$. The column $M_{j}$ represents the light distribution of the light that exits from segment $j$. Every element of the column 


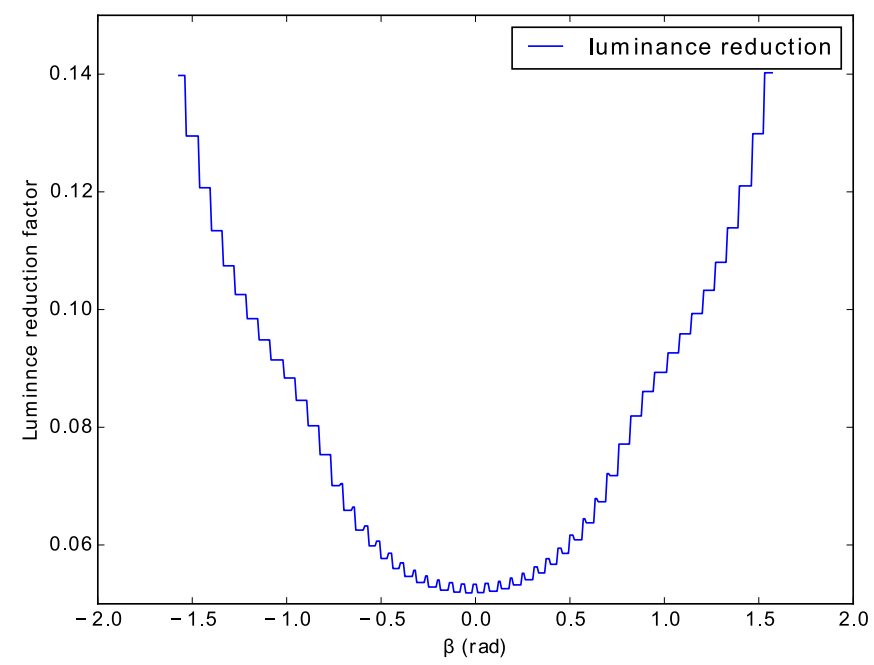

Figure 7: The reduction factor of the peak luminance compared to a smooth lens when viewed from a direction $\beta$ for a lens with 50 segments.

represents the flux emitted in a certain angular interval depending on the chosen resolution of the target distribution.

The elements of $M_{j}$ can be interpolated to form a continuous light distribution $M_{j}(\beta)$. The light distribution $S_{j}(\alpha)$ of the light that enters segment $j$ is equal to the input distribution $S(\alpha)$ over the angular domain $D_{j}$ of the considered lens segment.

The source distribution $S_{j}(\alpha)$ and the target distribution $M_{j}(\beta)$ are both normalized. In this way the light flux between input and output is conserved. Fresnel reflections are ignored which is a reasonable approximation since the angles of incidence on the lens surface are below the Brewster angle.

The lens surface can be generated by calculating a number of corresponding pairs $\alpha^{\prime}$ and $\beta^{\prime}$ on the cumulative distributions:

$$
\int_{\alpha_{j 0}}^{\alpha^{\prime}} S_{j}(\alpha) \mathrm{d} \alpha=\int_{\beta_{j 0}}^{\beta^{\prime}} S_{j}(\beta) \mathrm{d} \beta
$$

This equation expresses conservation of energy.

Every incident ray with an angle $\alpha^{\prime}$ must be refracted into an outgoing ray with an angle $\beta^{\prime}$. With the Snell's law of refraction, a surface normal $N$ can be calculated for every pair $\alpha^{\prime}$ and $\beta^{\prime}$. Starting from a fixed radius for each segment, the lens surface can be easily constructed by connection of consecutive line segments orthogonal to each surface normal.

There are two solutions for each lens segment. A concave solution and a convex solution. The convex solution can be obtained by a simple substitution $\beta \rightarrow-\beta$.

A resulting lens with 50 segments, for the example previously used in this section, can be seen in Fig. 1. 


\section{Example of a rotationally symmetric lens}

The design process for a rotationally symmetric 3D lens is in principle the same as for the case of a $2 \mathrm{D}$ lens. Below the light source, a rotationally symmetric illuminance $E(r)$ is desired. As an example we consider a Lambertian point source that is at a distance $D=3 \mathrm{~m}$ above the ground. We want to design a rotationally symmetric segmented lens for this source that generates an evenly illuminated area within a radius of $4 \mathrm{~m}$.

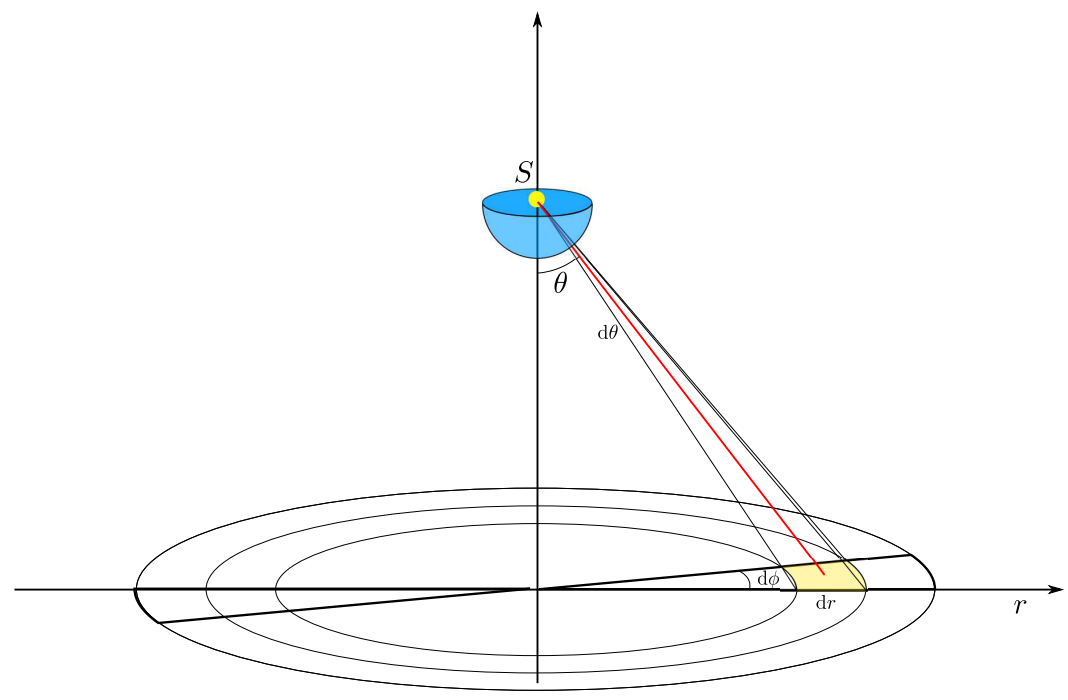

Figure 8: Schematic of the rotational symmetric situation. The lens contour is designed for a section $\mathrm{d} \phi$ and then rotated around the optical axis.

This is still a two dimensional design problem as it only requires the design of a cross section of the segmented lens which is then rotated around the optical axis. To get a two dimensional description of the problem, the illuminance is considered in an infinitesimal circle segment with opening angle $\mathrm{d} \phi$, as illustrated in Fig. 8. The light flux incident on an infinitesimal area $r \mathrm{~d} r \mathrm{~d} \phi$ must be equal to the light flux emitted in the solid angle $\mathrm{d} \theta \mathrm{d} \phi$ that encompasses this area. The relation between the illuminance $E(r)$ on a flat surface and the target intensity $T(\theta)$ in an angular section $\mathrm{d} \phi$ is described by:

$$
T(\theta) \mathrm{d} \theta \mathrm{d} \phi=E(D \tan (\theta)) \frac{D^{2} \tan (\theta)}{\cos (\theta)^{2}} \mathrm{~d} r \mathrm{~d} \phi
$$

Wherein the factor $\frac{D^{2} \tan \theta}{\cos (\theta)^{2}}$ represents the Jacobian for the transformation from the polar coordinate system on the target plane to angular coordinates.

Similarly, the input distribution $S(\theta)$ for a Lambertian source in a section $\mathrm{d} \phi$ is:

$$
S(\theta) \mathrm{d} \theta \mathrm{d} \phi=\frac{\Phi}{\pi} \cos (\theta) \sin (\theta) \mathrm{d} \theta \mathrm{d} \phi
$$

with $\Phi$ the value of the total luminous flux of the light emitted by the light source and $\sin \theta$ the Jacobian in the angular coordinates. The target and source intensities are shown in Fig. 9 The Jacobian factor in the rotational design is only exact in the case of a point source. Any spatial 
extent of the light source will cause a small but non-zero light flux towards the pole $=0$. Due to the rotational symmetry any non-zero contribution toward the pole will add up into a thin intensity peak in the direction of the pole. Because the total light flux in this peak is quite small, it suffices to add a slight surface roughness to the lens on the points where light is emitted towards the pole in order to reduce it.

The problem is thereby reduced to exactly the same situation as for a $2 \mathrm{D}$ lens wherein a source distribution $S(\alpha)$ should be transformed into a target distribution $T(\beta)$ The lens contour can be calculated as explained in Section 2 and rotated around the optical axis. The obtained lens is imported into Lighttools 8.3.2 and simulated. The resulting illumination pattern at a distance of $3 \mathrm{~m}$ as can be seen in Fig. 11 shows a uniform illuminance within a radius of $4 \mathrm{~m}$. A set of rays is depicted in Fig. 10 to illustrate the overlapping fans of rays generated by the freeform lens structures.

The presented lens illustrates the concept in the most simple manner. With a spherical inner surface refraction only happens at the segments of the outer surface. Other shapes of this first lens surface however are also possible. The extra refraction on the first lens surface will alter the shape of the lens segments of the outer surface which can improve the manufacturability of the design.

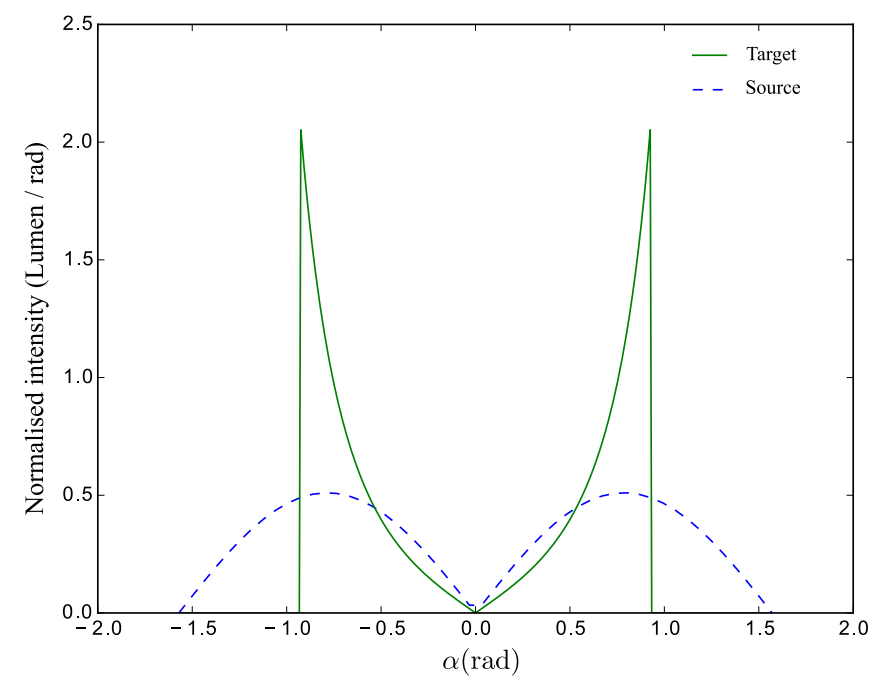

Figure 9: Representation of the Lambertian source distribution $S$ (blue) to be mapped onto the target distribution $T$ (green)

\section{Conclusion and Future work}

In this article, a novel method was presented to design a segmented lens with lateral or rotational symmetry that gives a much lower observed peak luminance compared to a smooth freeform lens. The segmented lens allows the same illuminance control as a smooth freeform lens with the same symmetry. By means of an iterative approach it is possible to tune the outgoing and overlapping light distributions from each lens segment so that all lens segments together transform a given input intensity distribution from a point source in a prescribed target intensity distribution. The overlapping light distributions of the different segments have the effect that the light flux from the 


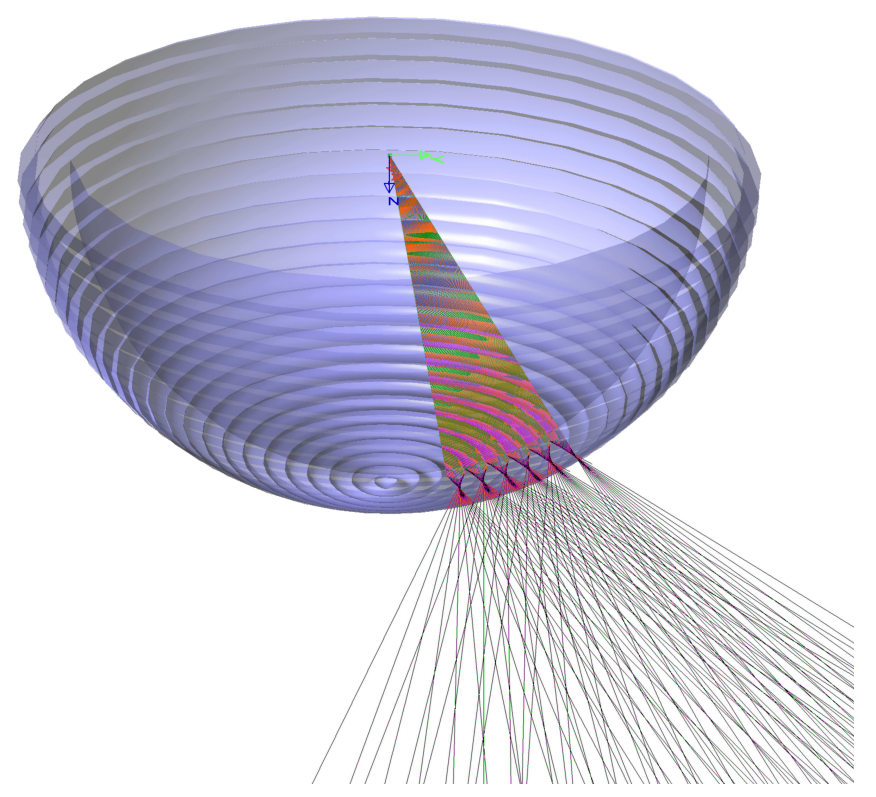

Figure 10: The rotationally symmetric segmented lens simulated in LightTools with a collection of rays to demonstrate the mixing effect of some structures
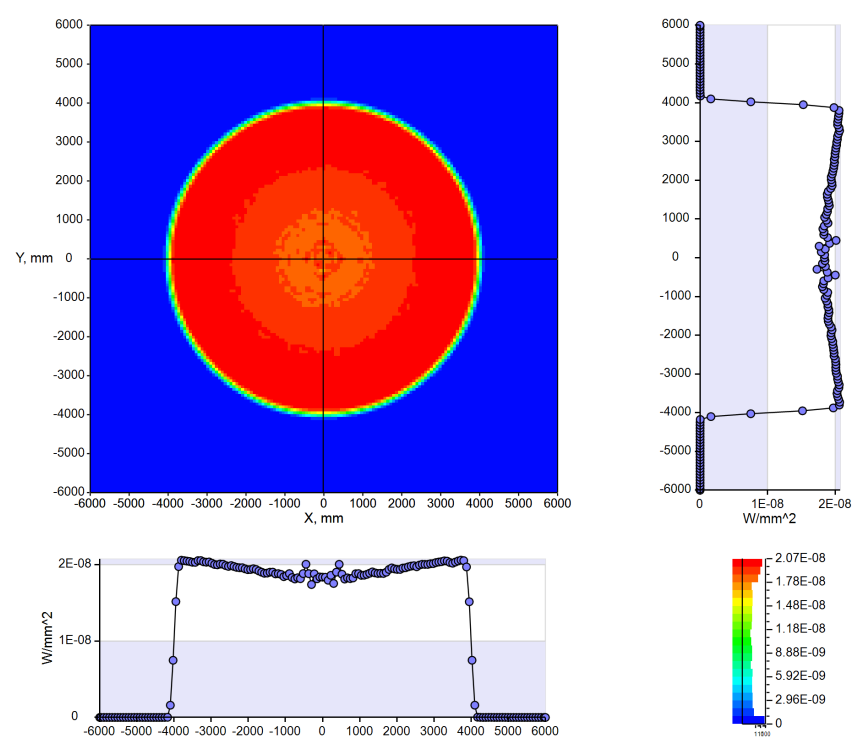

Figure 11: Simulated irradiance distribution on the target plane for the rotational symmetric lens in Lighttools 
light source reaches the eye via different paths trough the lens. The area on the lens surface from which light is emitted towards the eye thus increases while the light flux towards the eye remains the same as with a smooth lens. This effectively reduces the luminance compared with a smooth lens.

The method discussed here can be expanded in different ways. The great redundancy in variables adds the possibility of not only reducing the peak luminance but also tailoring the observed luminance across the lens according to specific requirements. Secondly the method can be expanded to 3-D lenses without lateral or rotational symmetry.

The solution for the transformation matrix $M$ that is obtained with the described iterative approach is only one of an infinite set of matrices that satisfy $\mathbf{T}=M \mathbf{S}$. There are of the order of $s \times t$ variables, depending on the maximum deviation angle $B$, that only have to satisfy $s+t$ equations. Namely the normalization condition of the $s$ columns and the set of $t$ equations $\mathbf{T}=M \mathbf{S}$. This leaves a degree of flexibility to further tailor the matrix $M$ to achieve specific requirements for the observed luminance from various observer positions. E.g. a uniform or smoot luminance pattern for certain observer positions in order to meet visual comfort requirements. This could be achieved just by adding another step in the algorithm that rescales the elements of each row.

The types of lenses considered in this publication are obtained by extrusion or rotation of a 2D lens contour. This has the convenience that the shape of the lens segments can be calculated from the transformation matrix. If unlike in section 3 there is also a variation of the target light distribution along the $\phi$-direction, it is no longer possible to obtain this target distribution by rotating $2 \mathrm{D}$ lens contour and a real segmented 3D freeform lens would be needed.

Such a 3-D lens would require the hemisphere around the light source to be divided into a tiling of $3 \mathrm{D}$ segments and the light fluxes trough these segments must be spread out in a similar way as described in this publication.

A similar algorithm as the one presented in this paper could be used to obtain a suitable intensity distributions for every lens segment in the 3D freeform array. The main difference is the fact that 2D intensity distributions should be used for both source and target distribution which will result in $4 \mathrm{D}$ transformation matrices.A further major difficulty however is that there is no simple way to derive the $3 \mathrm{D}$ lens surface in order to generate the required intensity distributions, as is in the 2D case. For each lens segment a complex numerical optimization step is needed. [Bösel and Gross, 2016][Bruneton et al., 2013b]

A relatively simple solution has been demonstrated by tiling a surface with identical freeform lenses [Aslanov et al., 2012]. This means that the luminance is spread over the complete lens surface, but requires that the incoming light distribution is the same for every lens and limits possible target output beams.

\section{Funding}

This research was supported by VLAIO (Flanders Innovation \& Entrepreneurship) through the O\&O project "Madonna: Methodologies and algorithms for the development and production of next-gens LED armatures (145023)".

\section{References}

Aslanov E, Doskolovich LL, Moiseev MA. 2012. Thin led collimator with free-form lens array for illumination applications. Appl Optics 51:7200-7205. 
Bösel C, Gross H. 2016. Ray mapping approach for the efficient design of continuous freeform surfaces. Optics Express 24:14271-14282.

Bruneton A, Bäuerle A, Wester R, Stollenwerk J, Loosen P. 2013a. High resolution irradiance tailoring using multiple freeform surfaces. Opt Express 21:10563-10571. doi:10.1364/OE.21.010563.

Bruneton A, Bäuerle A, Wester R, Stollenwerk J, Loosen P. 2013b. Limitations of the ray mapping approach in freeform optics design. Optics letters 38:1945-1947.

Feng Z, Froese BD, Liang R. 2016. Freeform illumination optics construction following an optimal transport map. Appl Optics 55:4301-4306. doi:10.1364/AO.55.004301.

Geerdinck L, Gheluwe JV, Vissenberg M. 2014. Discomfort glare perception of non-uniform light sources in an office setting. J Environ Psychol 39:5 - 13. Light, lighting, and human behaviour.

Helm R, Benoit R. 1990. Low-glare light. US Patent 4,969,075.

Rinehart T, Wood R, Purchase K. 2015. Light transmissive structures and fabrication methods for controlling far-field light distribution. US Patent 9,086,521.

Scheir GH, Hanselaer P, Bracke P, Deconinck G, Ryckaert WR. 2015. Calculation of the unified glare rating based on luminance maps for uniform and non-uniform light sources. Build Environ 84:60 - 67 .

Tashiro T, Kawanobe S, Kimura-Minoda T, Kohko S, Ishikawa T, Ayama M. 2015. Discomfort glare for white led light sources with different spatial arrangements. Lighting Res Technol 47:316-337.

Yamamoto S, Akao H. 2012. Lighting apparatus. US Patent App. 13/514,126. 